\title{
Helicobacter pylori in Rosacea: Epiphenomenon, Etiologic Agent or Maintenance Factor? Summary of 10 Years of Experience in Clinical Microbiological Practice
}

\begin{abstract}
Marcel Marcano-Lozada ${ }^{1,2 *}$
${ }^{1}$ Aggregate Professor Microbiology Department, "J.M. Vargas" School of Medicine, Universidad Central de Venezuela, Caracas, Venezuela ${ }^{2}$ Medical Microbiology Specialist, Medical Microbiology Unit, Angios Vascular Center and Wound Clinic, Caracas, Venezuela

*Corresponding Author: Marcel Marcano-Lozada, Aggregate Professor Microbiology Department, "J.M. Vargas" School of Medicine, Universidad Central de Venezuela, Caracas, Venezuela.
\end{abstract}

Received: September 04, 2019; Published: September 23, 2019

DOI: $10.31080 /$ ASMI.2019.02.0382

\section{Abstract}

A retrospective analysis of the clinical-microbiological records of the patients referred to the Medical Microbiology Unit (Angios Vascular and Clinical Wound Center, Caracas, Venezuela) was carried out over a period of 10 years (April 2007 - April 2017), with rosacea diagnosis refractory to conventional therapy. 124 records were recovered that met the "rosacea with poor response to topical and systemic therapy" inclusion criteria, and the microbiological data on infectious status by Helicobacter pylori (H. pylori) and clinical records were extracted and included in the Investigator Global Assessment (IGA) and Clinician`s Erythema Assessment (CEA) scales, classifying them according to the subtype of rosacea diagnosed and proceeding to the analysis, interpretation and discussion of the information obtained. Demographically, the female sex prevailed (120 patients - 97\%-), with an average age of 32,5 years, mainly Fitzpatrick's proto types II and IV (49 and 52 patients respectively), and subtype 2 of rosacea (79\%), history of previous therapy (systemic and topical) to control demodicosis in all patients, anti-infective therapy (topical and systemic) to control Cutibacterium acnes in 118 patients. The status of active gastric infection by H. pylori was demonstrated by the breath test with carbon 14 (UBT- C14) in 99 patients (79.84\%), predominantly in subtype 2 (90 patients - 90.91\% -); IGA values prior to eradication treatment against H. pylori, were mostly between grades 3 and 4 (78 and 11 patients respectively in subtype 2 ) and in the case of CEA, predominantly grade 3 (86 subtype patients 2). After administering the triple eradication therapy adjusted to each patient, its efficacy was evaluated (after the period of antimicrobial clearance), and a total elimination of the pathogen was obtained in the new breath test, with very marked clinical improvements for patients in the subtype 2 with IGA Clear " 0 " in 78 patients and Almost Clear " 1 " in 12 patients ( $\mathrm{p}<0,0001)$ and CEA " 0 " (Clear) in 88 patients and " 1 " (Almost Clear) in 2 patients, $p<0,0001$ ), and improvement was also observed in some patients of subtype 1 where 6 patients ended up with "2" IGA $(p<0,05)$. Additionally, elimination of gastric symptomatology (heartburn) was verified in all treated patients. Due to the retrospective nature of the study, treatment satisfaction could not be assessed. According to the results obtained, the eradication of active gastric infection by $H$. pylori, is associated with clinical improvement of rosacea, mainly in subtype 2, with clear and almost clear scores for IGA and CEA, although a discrete is also observed favorable effect in patients with subtype 1 , allowing to conclude on this analyzed data, a possible role of $H$. pylori as a trigger or cause of the maintenance of rosacea symptoms, especially of the inflammatory variant.

Keywords: Rosacea; Helicobacter pylori; Treatment Failure; IGA; CEA

\section{Introduction}

A non-dermatologist physician writing about rosacea is not frequent in the literature, much less evaluating therapeutic responses, but in the case of the medical microbiologist, the reference of patients with failures to conventional therapies in search of possible infectious triggers or factors that end up perpetuating the dermatological condition allows this article to be presented. The role of the medical microbiologist is to give diagnostic support to colleagues from different specialties, and in the case of dermatologists who face patients with rosacea refractory to treatment, it goes beyond verifying demodicosis, or intervening in the proliferation of Cutibacterium acnes (C. acnes -formerly Propionibacterium acnes-), reaching the identification by non- invasive methodologies of the presence of active gastric infection by Helicobacter pylori as proposed in the nineties [1], as a controversial agent within the pathophysiology and management of rosacea.

In Venezuela, one of the most frequent causes of reference from the dermatological consultation to the Medical Microbiology unit, only surpassed by superficial mycoses, chronic skin ulcers and skin and phone infections resistant to conventional therapy, is rosacea, but not just any rosacea, but subtype 2 or papulopustulose [2], with an important prevalence that can be up to $10 \%$, predominantly in the female sex and which according to numerous specialists should be considered a condition instead of a pathology [3].

Beyond vasomotor alterations, imbalance in innate and acquired immunity, environmental and food influences, exacerbated response to Demodex folliculorum, relationship with distress, 
among other factors, gastric infection by Helicobacter pylori [4,5], and modulation of the cutaneous and intestinal microbiome [6,7], have gained interest in understanding the onset and maintenance of the pathology, and especially in the therapeutic management of papulopustulose rosacea.

There are many theories that present rosacea as an extra gastric manifestation of Helicobacter pylori infection, similar to what has happened in the case of idiopathic chronic urticaria [8,9], where it went from being considered as an epiphenomenon to verify an important role as initiator or maintenance factor of chronic inflammatory responses, which when modulated or eliminated by eradication therapy with the elimination of gastric infection, significant clinical improvements and healing of dermatological pathology are observed [10-13]. That is why, at present, several authors have gathered evidence of the importance of making the proper diagnosis of active gastric infection by Helicobacter pylori in patients with rosacea, and consider its eradication therapy as part of its thera- peutic protocol, as it is associated with clinical improvement of skin pathology [14-17].

Considering the high prevalence of rosacea $(2-10 \%$ population), and the importance of achieving control to improve the quality of life of patients (mainly women) [3], because rosacea is a chronic disease, which increases its incidence with age and with a complex pathogenesis (vasomotor alterations, innate and acquired immunity, environmental and dietary influences, unbalanced response to dermal parasitosis), with a diverse clinical spectrum (subtypes) and with different responses to conventional therapeutics. According to the classification of the Committee of Experts of the National Rosacea Society (NRSEC), there are 4 subtypes of rosacea, listed in Table A [2]:

It is of particular interest of the author the fact that the majority of patients referred for the microbiological investigation of pathogens associated with rosacea, present an adequate microbiological

Table A: Rosacea Subtypes Classification according to the Committee of Experts of the National Rosacea Society [2]

\begin{tabular}{|c|c|c|c|}
\hline $\begin{array}{l}\text { Rosacea } \\
\text { subtype }\end{array}$ & Epidemiology & Clinical manifestations & Dermatopathology \\
\hline $\begin{array}{l}\text { Erythematous } \\
\text { telangiectatic } \\
\text {-RET- (I) }\end{array}$ & $\begin{array}{l}\text { Most frequent } \\
\text { presentation }\end{array}$ &  & $\begin{array}{ll}\text { - } & \text { Capillary and venule hyperplasia (upper } \\
\text { dermis) } \\
\text { - } \\
\text { - } \\
\text { Pelangiectasias } \\
\text { tiocytic infiltrate } \\
\text { - } \\
\text { Edematous dermis } \\
\text { Solar elastosis }\end{array}$ \\
\hline $\begin{array}{l}\text { Papulopustu- } \\
\text { lose } \\
\text {-RPP- (II) }\end{array}$ & $\begin{array}{l}\text { Second most } \\
\text { frequent pre- } \\
\text { sentation }\end{array}$ & $\begin{array}{l}\text { - } \quad \text { Papules and/or centrofacial erythema- } \\
\text { tous pustules } \\
\text { - } \quad \text { Centrofacial erythema } \\
\text { - Hard or soft centrofacial edema }\end{array}$ & $\begin{array}{l}\text { - Parakeratosis, exocytosis, acanthosis and } \\
\text { inflammatory cells in the epidermis. } \\
\text { - Papules: perivascular and perifollicular in- } \\
\text { flammatory infiltrate (lymphoplasmocytic } \\
\text { and neutrophilic in superficial and middle } \\
\text { dermis) } \\
\text { Pustules: neutrophilic surface accumula- } \\
\text { tion }\end{array}$ \\
\hline Fimatous (III) & $\begin{array}{l}\text { Higher fre- } \\
\text { quencys in men }\end{array}$ & $\begin{array}{l}\text { Erythematous and edematous skin } \\
\text { thickening } \\
\text { - } \begin{array}{l}\text { Proliferation of fibrous tissue and } \\
\text { sebaceous glands. }\end{array} \\
\text { Accenting of follicular orifices with } \\
\text { sebumand keratin plugs with unctu- } \\
\text { ous and smelly material }\end{array}$ & $\begin{array}{l}\text { - Initial stage: inflammation } \\
\text { Late-stage: Fibrosis, hyperplasia/seba- } \\
\text { ceous hypertrophy, inflammatory detritus } \\
\text { and telangiectasias }\end{array}$ \\
\hline Ocular (IV) & $\begin{array}{l}\text { Affects up } \\
\text { to } 50 \% \text { of } \\
\text { patients with } \\
\text { rosacea }\end{array}$ & $\begin{array}{l}\text { - } \quad \text { May precede skin lesions in } 20 \% \text { cases } \\
\text { - } \\
\text { - }\end{array}$ & - Unspecific \\
\hline
\end{tabular}


response to topical and systemic therapies to control the amount of $D$. folliculorum, with significant reduction in the count of this organism, mainly in patients with rosacea subtype 2, moreover, they are referred to the Medical Microbiology Unit to continue the search for possible infectious agents that participate in the genesis and / or maintenance of the pathology, and by particular facilities of the unit, these patients are temporarily delegated to the diagnosis, treatment and care of the microbiologist.

It is here that the investigation of the active gastric infection by the Gram-negative bacillus $H$. pylori is of great interest, since its pathogenesis at an extra gastric level is associated with chronic Th2 - type inflammatory responses such as that observed in rosacea, but nevertheless, there is controversy with the role played by $H$. pylori. Thus, rosacea has been linked to various digestive disorders, such as hypochlorhydria or achlorhydria, gastritis and changes in the jejunal mucosa. Periodic outbreaks of rosacea and peptic ulcer are often simultaneous, which seems to establish a relationship between the two phenomena. That way, rosacea could be an extra gastric sign of $H$. pylori infection.

Although the studies are inconclusive, some hypotheses have been suggested about the possible pathogenic role of $H$. pylori in rosacea. Flushing can be explained in the case of $H$. pylori by the direct or indirect production of potent vasoactive substances such as histamine, prostaglandins, leukotrienes, various cytokines, plasma gastrin and nitric oxide [4,5].

The objective of this study is to try to document the importance for the doctor who evaluates or manages patients with rosacea, to go beyond the treatment of demodicosis or to try to regulate Cutibacterium acnes, and find a deeper genesis to the chronic inflammatory process rooted deeper than in the skin, and therefore seeks to establish the need for research for $H$. pylori and its treatment, since the benefits end up being large and unsuspected as it is intended to demonstrate here.

Goal

To evaluate the role of eradication of active gastric infection by Helicobacter pylori in the clinical evolution of rosacea with poor response to conventional treatment (topical and/or systemic).

\section{Materials and Methods}

A retrospective review of the medical-microbiological histories was carried out in the period from April 2007 to April 2017, of those patients referred to the Medical Microbiology Unit for their microbiological evaluation and consequent medical treatment (more than 750 medical records with a diagnosis of rosacea were initially reviewed) and of these, 124 were selected that met the criteria for rosacea resistant or refractory to conventional therapy topical and systemic, and presented sufficient data to complete the clinical Scales (Investigator Global Assessment -IGA- \& Clinician's Erythema Assessment -CEA-), as well as having at minus 1 follow-up visit (control), and having been the object of serological investigation and active gastric infection by $H$. pylori, and having documented the use of triple eradication therapy in positive cases, as well as post-microbiological and clinical control treatment, to allow further analysis.

The IGA and CEA instruments contains 5 grades, that runs from 0 to 4 , summarized in Tables B \& C:

Table B: Investigator Global Assessment [18]

\begin{tabular}{|l|c|c|c|}
\hline Outcome & Grade & Score & Clinical description \\
\hline Treatment & Clear & 0 & $\begin{array}{c}\text { No inflammatory lesions } \\
\text { present, no erythema }\end{array}$ \\
\cline { 2 - 4 } Success & $\begin{array}{c}\text { Almost } \\
\text { Clear }\end{array}$ & 1 & $\begin{array}{c}\text { Very few small papules/ } \\
\text { pustules, very mild erythema }\end{array}$ \\
\hline $\begin{array}{l}\text { Treatment } \\
\text { Failure }\end{array}$ & Mild & 2 & $\begin{array}{c}\text { Few small papules/pustules, } \\
\text { mild erythema }\end{array}$ \\
\cline { 2 - 4 } & Moderate & 3 & $\begin{array}{c}\text { Several small or large papules/ } \\
\text { pustules, moderate erythema }\end{array}$ \\
\cline { 2 - 5 } & Severe & 4 & $\begin{array}{c}\text { Numerous small and/or large } \\
\text { papules/pustules, severe } \\
\text { erythema }\end{array}$ \\
\hline
\end{tabular}

Table C: Clinician's Erythema Assessment [19].

\begin{tabular}{|l|l|}
\hline \multicolumn{2}{|c|}{ Redness Intensity } \\
\hline Score & \multicolumn{1}{c|}{ Description } \\
\hline 0 & Clear with no signs of erythema \\
\hline 1 & Almost clear; slight redness \\
\hline 2 & Mild erythema; definite redness \\
\hline 3 & Moderate erythema; marked redness \\
\hline 4 & Severe erythema; fiery redness \\
\hline
\end{tabular}

All data and variables collected were organized in Microsoft Excel for Mac spreadsheets and plotted to facilitate their assessment. Statistical significance checking was performed using Fisher's exact test (statistical significance, $p<0,05$ )

Diagnosis of active gastric infection by $H$. pylori was made using the Urea Breath Test C14, and breath samples for the diagnosis were collected at first visit and in the follow up visit (at least 2 weeks after finish the proton pump-inhibitor treatment course), using a commercial Urea Breath Test C14 kit (Pytest ${ }^{\circledR}$, Ballard Medical Products, Lone Park, USA). Positive values were considered up to 200 DPM (Disintegrations per Minute), values between 51 - 199 DPM were considered indeterminate (repeat the test) and negative results were considered when the value was $\leq$ to $50 \mathrm{DPM}$ ), all patients positive for infection they should have been treated with one of the triple eradication therapy schemes for H. pylori, document the result to correlate with the expected variations of IGA and CEA. Serological history (IgM and IgG anti-H. pylori) was reviewed and recorded, as well as the presence of heartburn as a guiding symptom of ulcer-peptic disease, and these data were tabulated together. 
Helicobacter pylori in Rosacea: Epiphenomenon, Etiologic Agent or Maintenance Factor? Summary of 10 Years of Experience in Clinical Microbiological Practice

Tabulations and graphing of the breath test results were performed in contrast to the IGA and CEA values, at zero (baseline) time and then post-eradication therapy in patients who tested positive for UBT - C14 and received treatment.

\section{Results}

The main demographic characteristics of the population are summarized in table 1.

Table 1: Demographic characteristics of 124 patients diagnosed with rosacea with poor response to topical and systemic therapy. Medical Microbiology Unit, Caracas, Venezuela. 2007-2017.

\begin{tabular}{|c|c|}
\hline Characteristic & Patients date \\
\hline Sex & 120 female / 4 male $(96,8 \%$ / 3,2\%) \\
\hline Age & 32,5 years (range 19 - 46 years) \\
\hline \multirow{6}{*}{ Fitzpatrick's phototypes } & I $\quad 8 \quad(6,5 \%)$ \\
\hline & II $\quad 49(39,5 \%)$ \\
\hline & III $10(8,1 \%)$ \\
\hline & $52(41,9 \%)$ \\
\hline & $\begin{array}{lll}\mathrm{V} & 5 & (4 \%)\end{array}$ \\
\hline & VI \\
\hline \multirow{3}{*}{ Rosacea subtype } & $1 \quad 18(14,5 \%)$ \\
\hline & $298(79 \%)$ \\
\hline & $38(6,5 \%)$ \\
\hline $\begin{array}{l}\text { Therapeutic } \\
\text { history against } \\
\text { D. folliculorum }\end{array}$ & 124 / $124(100 \%)$ \\
\hline $\begin{array}{l}\text { Therapeutic } \\
\text { history against } C \text {. acnes }\end{array}$ & 118 / $124(95,2 \%)$ \\
\hline $\begin{array}{l}\text { Gastric symptoms } \\
\text { (pyrosis) }\end{array}$ & $102 / 124(82,3 \%)$ \\
\hline
\end{tabular}

The number of patients with gastric symptoms (pyrosis), their serological status for H. pylori acute (IgM) or past (IgG) infection, and their actual infectious status due to the results of the urea breath test with C14 (UBT - C14) for the whole population (124 patients) were summarized in Graphic 1.

The changes in the Investigator Global Assessment, in 99 patients with $H$. pylori active gastric infection were summarized compared pre (UBT - C14 positive) and post-treatment (UBT-C14 negative) in Graphic 2. It is necessary to mention that the triple therapy eradication schemes against $\mathrm{H}$. pylori infection were $100 \%$ effective, eliminating gastric infection in the 99 infected patients, this being verified by the post-treatment negative UBT - C14, and another interesting finding, the heartburn symptom disappear in all patients after the successful eradication therapy.

The variation in the Clinician's Erythema Assessment, in the 99 patients with $H$. pylori active gastric infection were summarized compared pre (UBT - C14 positive) and post-treatment (UBT - C14 negative) in Graphic 3.



Graphic 1: Gastric symptomatology, serological status and active gastric infection by $H$. pylori in 124 patients with treatment-resistant rosacea. Medical Microbiology Unit, Caracas, Venezuela. 2007-2017.



Graphic 2: Investigator Global Assessment and active gastric infection by H. pylori pre and post-treatment in 99 patients with treatment-resistant rosacea. Medical Microbiology Unit, Caracas, Venezuela. 2007-2017.

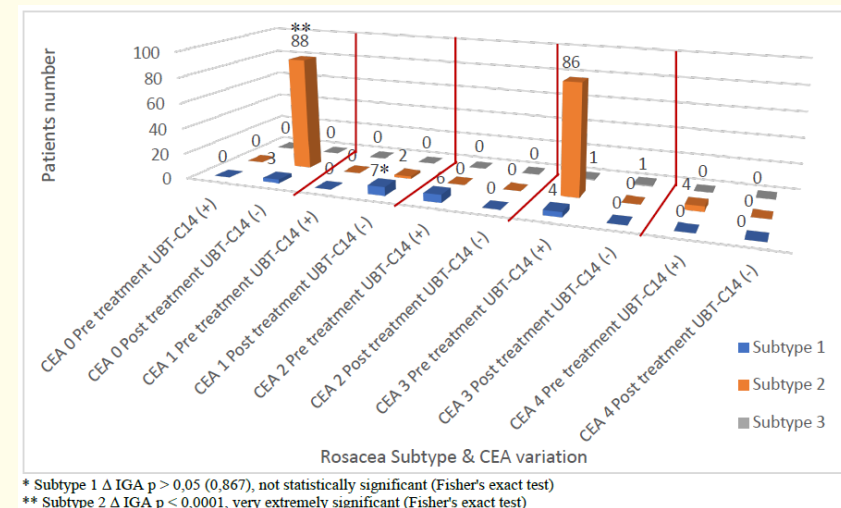

Graphic 3: Clinician's Erythema Assessment and active gastric infection by $H$. pylori pre and post-treatment in 99 patients with treatment-resistant rosacea. Medical Microbiology Unit, Caracas, Venezuela. 2007-2017. 


\section{Discussion}

As initially described, this group of patients whose data were collected adjusts the epidemiology of the globally described disease, where female sex predominates (only 4 stories of male patients were recovered) and youth (32.4 years of age) However, being Venezuela a country where miscegenation is important, the predominant Fitzpatrick's phototype was IV (52 patients), followed closely by phototype II ( 49 patients), and this can be motivated by the idiosyncrasy of Venezuelan female population, where the clearest skins to show more intense symptoms, seek medical help, but this inference of the search for resolution to a symptom that affects aesthetics, is equally valid for the phototype of darker skin.

Since these were patients referred to the Medical Microbiology Unit, the pathogens conventionally associated with exacerbations of rosacea (D. folliculorum and C. acnes) had been investigated in many cases, and therefore their modulation or attempted eradication is always recommended, This was verified by finding among the antecedents of previous therapy (systemic and topical), that all patients had been treated for demodicosis control, and antiinfective therapy (topical/systemic) had also been used to control C. acnes in almost all the patients (118/124 patients). However, although some of the medications used in the anti-infective therapy of rosacea may have some effect on H. pylori, they fail to modify the infectious burden or the possible chronic inflammatory responses associated with this Gram-negative bacillus.

These patients had not been initially screened for active gastric infection by $H$. pylori, although there was a high presence of gastric symptoms present, for example, in patients of subtype 2 (89 patients - 90.82\% -), and for In the Medical Microbiology Unit protocol, dermatologist and immunologist colleagues are asked to practice the investigation of active gastric infection, instead of only managing the concept of serological scar, since even high titers IgG anti-H. pylori are not associated with protection against infection, but with exposure to the pathogen. Although IgG serology was positive in all patients of subtype 2 (98/98), with the negative IgM correlate, this criterion was not taken as the determinant to indicate treatment, since seroprevalence in Venezuelan adults is between 75 to $95 \%$ [20], because the infection caused by bacteria occurs in early stages of life, preferring a more reliable criterion such as the demonstration of active gastric infection by $H$. pylori by means of the breath test with carbon-labeled urea 14 (UBT - C14), which by its non-invasive nature and high sensitivity and specificity, in addition to the quickly available result, allowed to really discriminate whether the infection could be immunomodulating a possible chronic inflammatory response of type Th2, to be repeated after therapeutic intervention to confirm if bacterial eradication was achieved, which was effective in $100 \%$ of the 99 patients with positive UBT - C14, and for which various schemes of triple eradication therapy were used ( 1 proton pump inhibitor for 6 weeks +2 anti- microbials combined for 7 to 14 days), which varied over 10 years, and were adjusted according to the international treatment guidelines, therapeutic and personal history of each patient, in addition to being guided by local epidemiology, thanks to the knowledge of antimicrobial resistance / susceptibility patterns of $H$. pylori on the basis of microbiological studies of gastric biopsy cultures and their antibiograms using the minimum inhibitory concentration (MIC) determinations by the E-test method [21]. The above allowed to select schemes where the use of Metronidazole is not included, which is still one of the main active drugs in the management of this infection, but which, in the Venezuelan case, has more than $70 \%$ resistance, which it is ineffective as an empirical therapy.

Understanding now that, after at least 6 weeks after the end of the anti-infectious treatment (which also translates into at least 2 weeks after the end of gastroprotection with proton pump inhibitors), a potential response focus is eliminated. chronic inflammatory (demonstrated by negativity of the UBT - C14), which has been or demonstrated as a possible causative agent in extra gastric pathologies [22], the most studied being spontaneous urticaria (previously idiopathic chronic) $[9,10,23]$ and in certain purples such as Henoch's Schoenlein [24] and thrombocytopenic idiopathic [25], in addition to the known causal associations in ulcer-peptic disease and gastric neoplasms (T-cell lymphoma associated with gastric mucosa and gastric adenocarcinoma) [26] there is evidence of improvement in these pathologies (and regression or cure), we can understand the changes related to clinical healing (IGA and CEA "Clear" or "Almost Clear) with very extreme statistical significance ( $p<0,0001$ ) after eradicating H. pylori, in patients of subtype 2 , it is necessary to consider within the routine work plan a patient with rosacea (and especially papulopustulose), the determination of active gastric infection by $H$. pylori and its consequent treatment, as had been raised for several years by some authors [27,28].

Although improvement in patients with rosacea telangiectasia subtype 1 also showed improvement, the nature of less symptomatic inflammation and greater vasodilation can explain why the benefit of eradication of active gastric infection by H. pylori, assumed as a chronic inflammation enhancing agent, is smaller in this subgroup of patients, and almost non-existent in the fimatous variant (subtype 3).

\section{Conclusion}

According to the results obtained in this 10 years retrospective evaluation, the eradication of gastric active infection by H. pylori, is associated with clinical improvement of rosacea verified by variation to the improvement of up to 3 degrees in the IGA and CEA, mainly in subtype 2 , with discreet favorable effect in patients with subtype 1 , establishing a possible role of $H$. pylori as a trigger or cause of the maintenance of rosacea symptoms, especially of the inflammatory variant. 


\section{Bibliography}

1. Rebora A., et al. "May Helicobacter pylori be important for dermatologists?". Dermatology 191.1 (1995): 6- 8.

2. Wilkin J., et al. "Standard classification of rosacea: report of the National Rosacea Society Expert Committee on the Classification and Staging of Rosacea". Journal of the American Academy of Dermatology 46.4 (2002): 584-587.

3. Troielli P., et al. "Update and recommendations for the diagnosis and treatment of rosacea in Latin America". Medicina Cutánea Ibero-Latino-Americana 44.1 (2016): S7-S26.

4. Hirschmann JV. "Does Helicobacter pylori have a role in the pathogenesis of rosacea?". Journal of the American Academy of Dermatology 42.3 (2000): 537-539.

5. Bamford JT., et al. "Does Helicobacter pylori eradication treatment reduce the severity of rosacea?". Journal of the American Academy of Dermatology 42 (2000): 535-536.

6. Dahl MV., et al. "Temperature regulates bacterial protein production: possible role in rosacea". Journal of the American Academy of Dermatology 50.2 (2004): 266-272.

7. Picardo M and Ottaviani M. "Skin microbiome and skin disease: the example of rosacea". Journal of Clinical Gastroenterology 48.1 (2014): S85-S86.

8. Marcano L MJ., et al. "Helicobacter pylori en dermatologia. Breve revision de la literature". Derm Venz 41.2 (2003): 3-7.

9. Marcano-Lozada M., et al. "Association among Helicobacter pylori Gastric Infection Eradication and Chronic Idiopathic Urticaria Improvement in Venezuelan Patients". Journal of Advanced Research in Microbiology 2 (2018): 004.

10. Marcano-Lozada M., et al. "Clinical response of idiopathic chronic urticaria to the eradication of Helicobacter pylori infection". Medicina Cutánea Ibero-Latino-Americana 35.3 (2007):13013-5.

11. Curth HM., et al. "Effects of Helicobacter pylori Eradication in Chronic Spontaneous Urticaria: Results from a Retrospective Cohort Study". American Journal of Clinical Dermatology 16.6 (2015): 553-558.

12. Mogaddam MR., et al. "Relationship between Helicobacter pylori and idiopathic chronic urticaria: effectiveness of Helicobacter pylori eradication". Postepy Dermatology and Allergology 32.1 (2015):15-20.

13. Persechino S., et al. "Chronic idiophatic urticaria and Helicobacter pylori: a specific pattern of gastritis and urticaria remission after Helicobacter pylori eradication". International Journal of Immunopathology and Pharmacology 25.3 (2012):765-770.

14. Jørgensen AR., et al. "Rosacea is associated with Helicobacter pylori: a systematic review and meta-analysis". Journal of the European Academy of Dermatology Venereology 31.12 (2017): 2010-2015.
15. Saleh P., et al. "Effects of Helicobacter pylori treatment on rosacea: A single-arm clinical trial study". Journal of Dermatology 44.9 (2017): 1033-1037.

16. Lazaridou E., et al. "Rosacea and Helicobacter pylori: links and risks". Clinical, Cosmetic and Investigational Dermatology 10 (2017): 305-310.

17. Yang X. "Relationship between Helicobacter pylori and Rosacea: review and discussion". BMC Infectious Diseases 18.1 (2018): 318.

18. Tan J., et al. "Updating the diagnosis, classification and assessment of rosacea: recommendations from the global RO Sacea CO nsensus (ROSCO) panel". British Journal of Dermatology 176.2 (2017): 431-438.

19. Tan J., et al. "Reliability of Clinician Erythema Assessment grading scale". Journal of the American Academy of Dermatology 71.4 (2014):760-763.

20. Cavazza ME., et al. "Helicobacter pylori infection in Venezuela". Clinical Microbiology and Infection 7.1 (2001): 331.

21. Urrestarazu MI., et al. "Susceptibility of Helicobacter pylori to antimicrobials". Rev Soc Ven Microbiol (2003): 14-15.

22. Hergueta P., et al. "Helicobacter pylori infection and extradigestive pathology". Annals of the Health System of Navarra 21.2 (1998).

23. Marcano-Lozada MJ., et al. "Eradication of Helicobacter pylori infection associated with clinical improvement of idiopathic chronic urticaria Preliminary results". Rev Panam Infectol 12.3 (2010): 22-27.

24. Reinauer S., et al. "Schönlein-Henoch purpura associated with gastric Helicobacter pylori infection". Journal of the American Academy of Dermatology 33 (1995): 876-879.

25. Kim BJ., et al. "Helicobacter pylori Eradication in Idiopathic Thrombocytopenic Purpura: A Meta-Analysis of Randomized Trials". Gastroenterology Research and Practice (2018): 6090878

26. International Agency for Cancer Research. Shistosomes, liver flukes and Helicobacter pylori. IARC Monographs on the evaluation of carcinogenic risks to human. IARC: Lyon (1994): 61.

27. Utas S., et al. "Helicobacter pylori eradication treatment reduces the severity of rosacea". Journal of the American Academy of Dermatology 40.3 (1999): 433-435.

28. Szlachcic A. "The link between Helicobacter pylori infection and rosacea". Journal of the European Academy of Dermatology Venereology 16.4 (2002): 328-336.

\section{Volume 2 Issue 10 October 2019 (C) All rights are reserved by Marcel Marcano-Lozada.}

